\title{
Peer-Assisted View-Dependent Progressive Mesh Streaming
}

\author{
Wei Cheng Dan Liu Wei Tsang Ooi \\ National University of Singapore \\ \{chengwe2, liudan, ooiwt $@ @$ comp.nus.edu.sg
}

\begin{abstract}
Progressive mesh streaming is increasingly used in 3D networked applications, such as online games, virtual worlds, and digital museums. To scale such applications to a large number of users without high infrastructure cost, we apply peer-to-peer techniques to mesh streaming. We consider two issues: how to partition a progressive mesh into chunks and how to lookup the provider of a chunk. For the latter issue, we investigated into two solutions, which trade off server overhead and response time. The first uses a simple centralized lookup service, while the second organizes peers into groups according to the hierarchical structure of the progressive meshes to take advantage of access pattern. Simulation results show that our proposed systems are robust under high churn rate, reduce the server overhead by more than $90 \%$, keep control overhead below $10 \%$, and achieve low average response time.
\end{abstract}

Categories and Subject Descriptors I.3.2a [Graphics Systems]: Distributed/Network Graphics; C.2.4b [Distributed Systems]: Distributed Applications

General Terms Performance, Design

Keywords 3D data, streaming, progressive meshes, peer-to-peer

\section{INTRODUCTION}

Advances in 3D scanning technology and 3D modeling techniques have enabled creations of huge, high-resolution, 3D meshes. These meshes are increasingly available for viewing over the Internet, in applications such as virtual worlds and virtual museums. Huge models may take long time to download completely for display at the client. For example, the Stanford model of the David statue, with 28 million vertices and 56 million triangles, is still 70 MB in size with state-of-the-art compression [3] and needs around 10 minutes to download at $1 \mathrm{Mbps}$.

To reduce waiting time, huge meshes are usually progressively coded [14] and transmitted via progressive streaming, where a low resolution version of the mesh (called base mesh) is transmitted and rendered first. Refinements (called vertex splits) are then continuously transmitted to incrementally improve the level of details

Permission to make digital or hard copies of all or part of this work for personal or classroom use is granted without fee provided that copies are not made or distributed for profit or commercial advantage and that copies bear this notice and the full citation on the first page. To copy otherwise, to republish, to post on servers or to redistribute to lists, requires prior specific permission and/or a fee.

MM'09, October 19-24, 2009, Beijing, China.

Copyright 2009 ACM 978-1-60558-608-3/09/10 ...\$5.00. of the mesh (See Figure 1). Each vertex split splits a vertex into two vertices. This technique allows the client to view a coarse version of the mesh quickly, trading off details and waiting time. The client may stop receiving the refinement once sufficient details are received. Further, only the visible region needs to be sent (so called view-dependent [15] streaming).

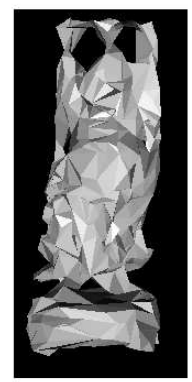

1000 vertices base mesh

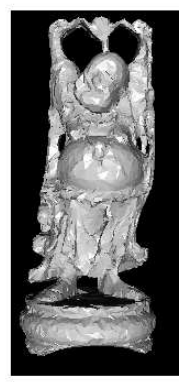

10000 vertices

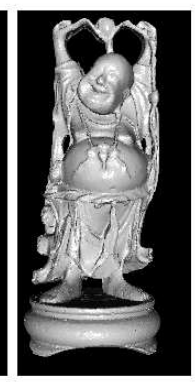

100000 vertices

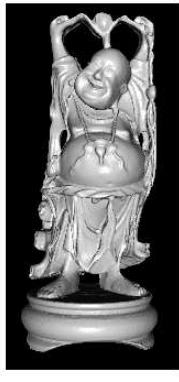

543652 vertices original mesh
Figure 1: Progressive 3D mesh streaming. Original mesh courtesy of Stanford Computer Graphics Laboratory.

In applications such as virtual art gallery, virtual earth, virtual museums, and virtual auction house, statues, artifacts, and auction items are streamed to potentially a large number of visitors or bidders, sometimes within a short period of time (e.g., when an item is released for bidding). The clients for these applications are likely to inspect the items carefully, zooming in to view the fine details, and rotating the item back-and-forth to examine all facets of an item. Such flash crowd can potentially impose substantial bandwidth requirements on the server.

Peer-to-peer (P2P) data dissemination is commonly used to alleviate server's bandwidth cost. Downloading peers forward the received data to other peers, contributing their upload bandwidth and reducing the burden of the server, hence allowing more users to be supported. P2P techniques have been successfully used in bulk file transfer (e.g., BitTorrent) and video streaming (e.g., PPLive).

Using P2P techniques for view-dependent progressive mesh streaming, or P2P mesh streaming for short, poses several new challenges. First, each client may view, and therefore request mesh data, in a different order. Thus, a client needs to frequently search for peers to download visible mesh data from when its view point changes. Second, we found that a user stays in the system for a short time, in the order of minutes (Figure 8(a)) when viewing a mesh, leading to high churn rate. Third, determining the visible region of a mesh given a view point is computationally intensive and is traditionally 
done at the server. In the $\mathrm{P} 2 \mathrm{P}$ context, how to determine the visible region of a mesh is non-trivial.

Fortunately, the nature of mesh streaming alleviates other constraints in the system design: (i) we found that users can tolerate higher response time when interacting with the mesh, due to progressive mesh rendering, and (ii) unlike video, there is no strict deadline to render the mesh. Together, these differences lead us to new challenges and different design decisions in designing $\mathrm{P} 2 \mathrm{P}$ mesh streaming systems, in contrast to $\mathrm{P} 2 \mathrm{P}$ video streaming and $\mathrm{P} 2 \mathrm{P}$ file downloading. This paper reports on our design of a P2P mesh streaming system and its evaluations.

In P2P data dissemination, the data (a file, video, or mesh) are typically divided into chunks. A peer that has downloaded a chunk can potentially serve this chunk to other peers. We call such a peer a provider of the chunk. This paper addresses two important design questions of P2P mesh streaming.

The first question is how to define a chunk. While one can easily segment a file or a video into chunks, due to progressivity of progressive meshes, chunks have to be carefully constructed in a hierarchical way that can incrementally improve the quality of the mesh, with minimal coding dependency among the chunks. Moreover, chunk size should be small enough to reduce the fraction of invisible vertex splits received.

Second, how can a peer know the best provider of a given chunk? Consider the a large number of queries and high churn rate, we first consider simply using a centralized lookup service for chunk provider, allowing fast peer failure detection and one-hop lookup. Centralized lookup, however, is not scalable to large number of peers, due to the overhead in maintaining peer states and handling queries. We therefore propose a hierarchical P2P system, which retains the advantages of centralized lookup but with significantly fewer requests to the server. The basic idea is to group peers according to the hierarchical structure of chunks in a progressive mesh. Each group has a leader that takes over most of the responsibilities of the server to reduce server overhead.

Our contributions in this paper are as follows. First, we compare and contrast $\mathrm{P} 2 \mathrm{P}$ mesh streaming to $\mathrm{P} 2 \mathrm{P}$ video streaming and $\mathrm{P} 2 \mathrm{P}$ file downloading and point out the main difficulties of $\mathrm{P} 2 \mathrm{P}$ mesh streaming. Second, considering the differences and challenges, we investigate on two content discovery schemes for P2P mesh streaming. The structure of the progressive mesh is considered in the second design to further reduce server overhead. Third, we propose a chunking scheme for progressive meshes to support P2P mesh streaming. Finally, we run simulations based on real traces collected from users to evaluate the P2P mesh streaming system we proposed. Analysis and simulation results show that server overhead can be reduced by $90 \%$. Meanwhile, average response time and control overhead are low.

We structure the rest of the paper as follows. We introduce previous work on progressive mesh streaming and $\mathrm{P} 2 \mathrm{P}$ techniques in Section 2. We compare P2P mesh streaming with P2P video streaming and P2P file downloading in Section 3. Sections 4 and 5 introduces the receiver-driven protocol and the hierarchical chunk structure, two essential components of our system, respectively. We propose two content discovery schemes in Section 6. We present experimental results in Section 7 and conclude in Section 8.

\section{RELATED WORK}

\subsection{Streaming of 3D Data}

Previous studies in 3D data streaming have considered different approaches for data representation, including point-based, image- based, and mesh-based. In this section, we focus on mesh-based representation as it is most related to our work.

The main concern in streaming of 3D meshes is how to improve the quality of the received mesh as fast as possible, mitigate distortion to rendered mesh in the presence of packet losses, and scale to a large number of users.

At the transport layer, Al-Regib and Altunbasak [1], Li et al. [19], and Chen et al. [7] have investigated how to intelligently select either TCP or UDP for transmissions to trade off reliability and end-to-end delay. Li et al. have also considered SCTP with partial reliability. Harris III and Kravets [13] propose a new transport protocol that exploits loss tolerance and partially-ordered property of 3D objects organized into trees of bounding volumes.

At the application layer, the major error control techniques: error resilient coding, error protection, retransmission, and error concealment, have all been applied to mesh streaming. Park et al. [23] and Yan et al. [25] focus on how to best segment a progressive mesh into smaller partitions such that it is more resilient to losses. Al-Regib et al. [2] consider a joint-source channel coding method to determine appropriate level of error protection to use. Chen et al. [7] also use FEC for transmission of 3D data. Error concealment is considered by Park et al. [22]. Cheng et al. [9] argue for retransmissions as the main error control method, while Tian [24] uses selective retransmission in their system.

The issue of scaling 3D streaming to a large number of users has not been sufficiently addressed. In our previous work, we focus on reducing the computational overhead at the server [8], by shifting the burden of visibility computation from the sender to the receiver. Hu et al. [16] and Cavagna et al. [6] have considered using peer-topeer architecture to stream a 3D scene to improve scalability. Our work is similar in spirit, but we focus on streaming single, large, progressive mesh in a view-dependent manner, rather than a 3D scene, where visibility decision is mainly done at the object level. Streaming progressive meshes needs a much finer granularity for view dependency. In this paper, we study view-dependent streaming at chunk level, which can be of much smaller size (can be as small as a packet) than an object.

Yang et al. [26] proposed a view-dependent 3D video streaming system, in which a set of gateways is used to disseminate the streams depending on the views of the receivers. Similar to our goals, the technique aims to reduce the server overhead. The number of gateways, however, is limited and the gateways are assumed to be stable. Further, 3D videos are not progressive in nature.

\subsection{P2P Video Streaming}

$\mathrm{P} 2 \mathrm{P}$ techniques have been widely studied in file downloading, live streaming, and video-on-demand (VoD) streaming applications. In this section, we introduce the related work on P2P video streaming systems.

Generally, P2P streaming systems can be categorized into treebased and mesh-based approaches. In tree-based approaches, peers are organized into a single tree $[17,10]$ or multiple trees $[5,21]$. In typical multi-tree-based approaches, like CoopNet [21], different parts of a video segment are separately pushed down along different sub-trees. SplitStream [5] utilizes Multiple Description Coding (MDC) scheme to further improve streaming quality by introducing data redundancy. Besides, each peer serves as an interior node in only one sub-tree to minimize the negative effect of node failures.

To further improve robustness and scalability, mesh-based approaches have been more widely explored in $\mathrm{P} 2 \mathrm{P}$ live streaming systems, including PPLive and PPStream. Typically, gossip-like protocols are used for peers to pull video content from qualified peers. Compared to tree-based approaches, such systems incur 
higher end-to-end latency and control overhead. To reduce these negative effects, push-pull-based approaches are proposed in [28]. Moreover, PRIME [20] incorporates swarming scheme and MDC to improve robustness and streaming quality, but its two-phase design increases end-to-end latency.

Similar tree-based and mesh-based approaches can also be utilized in P2P VoD streaming. Different from live streaming, peers may seek to different playback points in the video, causing higher peer dynamics. Further, since peers have different views of the video, it is more challenging to locate providers. The oStream [11] system utilizes sliding window to maintain certain video content so that peers can obtain content from other peers with smaller interval between their windows. Yiu et al. [27] propose that each peer randomly stores some segments and uses DHT to locate peers storing previous, current, and next segments, respectively. This approach can achieve better scalability and shorter initial delay than sliding window scheme, but maintaining the segment lists in a highly dynamic environment is difficult.

\section{P2P MESH STREAMING}

Although P2P techniques have already been studied in file downloading, live video streaming, and video-on-demand streaming, P2P view-dependent progressive mesh streaming (or P2P mesh streaming for short) has a different set of requirements and characteristics, leading to different design decisions and new challenges. In this section, we elaborate on these differences.

In $\mathrm{P} 2 \mathrm{P}$ file sharing, a peer is interested in obtaining a complete file. In most cases, the file is not useful until it is completely downloaded. During downloading, any new chunk of the file downloaded is useful. Chunks can be downloaded in any order. Therefore, peer $p$ can receive chunks from peer $q$ as long as $q$ possesses fresh chunks that $p$ have not received.

In $\mathrm{P} 2 \mathrm{P}$ video streaming, however, the video is played back mostly in the increasing time order ${ }^{1}$. The point the peer is watching the video is the playback time, and each chunk in the video has an associated timestamp. Unlike file sharing, not every chunk is useful. A peer is only interested in a chunk whose timestamp is later than the playback time. Chunks nearer to the playback time have higher priority. Since chunks are needed and playback in the same order, if a peer $p$ receives a chunk with timestamp $t$ from a peer $q$, it is likely that $p$ can receive the chunk with timestamp $t+1$ from $q$. In other words, a peer can exploit temporal locality in chunk access to discover other peers to retrieve the chunks from.

Temporal locality does not apply to mesh. In P2P mesh streaming, however, users might be interested in different parts of the mesh at different level of details. Each may choose to look at different facets, or zoom into different levels. Therefore, each peer may be interested in different chunks of the mesh at different time. Consequently, even if peer $p$ can receive a chunk from peer $q$ now, $p$ may not receive subsequent chunks from $q$ later, since $p$ may request a chunk that $q$ has never seen and is not visible to $q$. Thus, a peer would have to continuously query for peers to retrieve the mesh data from, as the peer's view point and level of details change over time. More queries are needed in mesh streaming than in file downloading or video streaming. Reducing such overhead is one of the main challenges of $\mathrm{P} 2 \mathrm{P}$ mesh streaming.

Another characteristic of mesh streaming is that the session length, i.e. how long a user stays in the system, is significantly shorter than that in file downloading and video streaming. Users usually leave the system after viewing all the interesting parts of a mesh in several minutes. Such short session time increases the churn rate and

\footnotetext{
${ }^{1}$ unless the user seek to another playback point
}

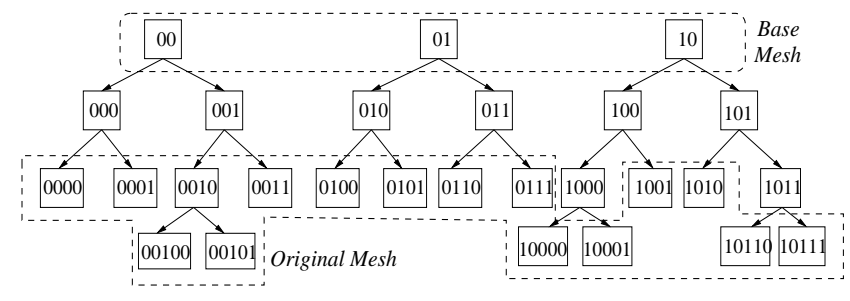

Figure 2: Vertex hierarchy. A rectangle represents a vertex and the number inside is its identification number, including tree ID and node ID.

makes typical approaches to reduce query overhead inappropriate. To reduce the number of queries, a peer typically caches information about the chunks available in a provider for future requests. High churn rate invalidates these cache information quickly.

\section{RECEIVER-DRIVEN MESH STREAMING}

To exposit our $\mathrm{P} 2 \mathrm{P}$ view-dependent mesh streaming system, we first address the question of the visibility determination. Traditionally, view-dependent mesh streaming systems use sender-driven protocols, in which the receiver sends the view point to the sender. The sender computes which vertex splits are visible and sends the vertex splits in the decreasing order of the visual contributions to the receiver. The sender also maintains the states of which vertex splits have been sent for each receiver, to avoid sending duplicate data. This design is originally meant for client/server architecture. In $\mathrm{P} 2 \mathrm{P}$ mesh streaming, a peer plays the role of the sender. This design is not desirable for P2P streaming for three reasons. First, computing the visibility and sorting the vertex splits are expensive and may deter peers from participating. Second, a peer might not have the complete mesh so determining visibility may not be possible. Finally, maintaining the receiver states requires synchronization across multiple peers, since a peer might retrieve data from multiple other peers.

We have previously [8] proposed a receiver-driven protocol, in which a receiver determines the visible vertices based on its own view point and explicitly requests these vertex splits. The sender, under this protocol, is stateless and simply serves the requests. We adapt the protocol in this work, as its stateless server design fits naturally into P2P mesh streaming.

The key question in designing the receiver-driven protocol is how a receiver can determine the visible vertices when it has not yet received the complete mesh. In our proposal, we exploit the progressive nature of meshes and assume that if a vertex is visible, the vertex from which it is split is also visible. Therefore, we split visible vertices to refine the mesh. Our experiments found that we can approximate the visible vertices with negligible errors using this rule of thumb [8].

Once a receiver finds a visible vertex, it requests the vertex split from the sender to split this vertex. To allow the receiver to explicitly request a vertex split, a unique identification (ID) is assigned to each vertex split. In a progressive mesh, vertex splits are hierarchically organized as a forest of binary trees, with a parent vertex $v$ being a coarser representation of its two children. Thus, the leaves of the trees are vertices belonging to the original mesh (the mesh before simplification). The roots of the trees are the vertices in the base mesh (See Figure 2).

We use the method proposed by Kim and Lee [18] and assign each vertex a bit string consisting of two parts - a tree ID and a node ID. The tree ID is the sequence number of the root of this tree 
in the base mesh, and the node ID represents the path from the root to this vertex in the binary tree. For example, if the tree ID is ' 01 ', which is also the ID of the root of this tree, the bit string ' 010 ' and ' 011 ' are the IDs of the left and right child of the root respectively. Figure 2 shows an example of vertex hierarchy.

Since the IDs embed the vertex hierarchy, all IDs can be deduced. The ID of the vertices in the base mesh is their sequence number, and the IDs of new vertices generated from a split can be deduced from the parent's ID. Thus, the sender need not send additional information to identify a vertex.

\section{HIERARCHICAL CHUNK STRUCTURE}

We now elaborate on how to group vertex splits into chunks while maintaining progressiveness among the chunks. In the original design of receiver-driven protocol [8], the receiver explicitly requests individual vertex splits. Such design is not appropriate for P2P streaming for three reasons. First, the receiver needs to send one ID ( 2 bytes in our implementation) to request for one vertex split (less than 5 bytes in our implementation). Hence, the requests occupy a large proportion of the up-link bandwidth, which is precious in P2P streaming, in which the up-link bandwidth are needed to share data with other peers. Second, data packets need to be generated dynamically at the sender whenever requests are received, increasing computation overhead and delay. Finally, a peer needs to find proper peers to retrieve each vertex, which is expensive since the number of vertices is huge in a large progressive mesh.

To address these drawbacks, we adapted the receiver-driven protocol to the concept of chunk, commonly used in P2P systems. Our chunk, however, is of much finer granularity, to allow a peer the flexibility of retrieving only the mesh data within current visible region. Each chunk consists of multiple vertex splits, and each vertex split only belongs to one chunk. When a peer needs a vertex split, it finds the chunk that includes this vertex split and sends its chunk ID to request it. Each chunk is requested only once to avoid duplicate requests. Once a chunk is received, all the vertex splits in this chunk are decoded and processed.

This method sacrifices some flexibility in choosing vertices, but has several advantages. First, it significantly reduces the up-link bandwidth requirement since now only one chunk ID is needed to request a set of vertices. Second, the cost of searching for peers to retrieve data is also reduced. Third, grouping vertex splits into chunks can be done offline at the server, so no computation cost and time are needed by peers for online packetization.

The question now is that how a peer knows which chunk to request when it decides to refine a certain part of a mesh. One naive solution is to store the chunk ID together with vertex splits, but this method adds relatively expensive overhead to each vertex split. For example, the chunk ID usually accounts for 2 bytes, while each encoded vertex split accounts for only 3-4 bytes, so the overhead is more than $50 \%$. Another method we considered is to organize the mesh into many bounding boxes with the same size, and the vertex splits of the vertices inside a box belongs to a chunk. The chunk ID can thus be deduced from the vertex coordinates. Nonetheless, this method still needs extra information to associate the chunks with the bounding box. Moreover, since a vertex in a bounding box might have ancestors in other bounding boxes, this method increases dependencies among chunks, which might delay the decoding of some received vertices until other chunks are received.

In our solution, we define chunks based on vertex dependencies as follows. First, we simplified the mesh to $s$ vertices. For convenience, we choose $s$ to be the power of 2, i.e., $s=2^{i}$. Then we split these vertices up to $w$ levels and use the generated mesh as the base mesh. During the split, each of the original $s$ vertices is

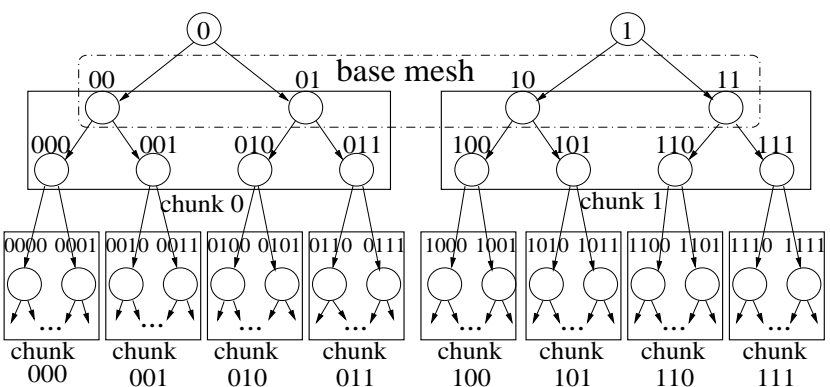

Figure 3: An example of hierarchical chunk structure. Here $w$ $=1, d=2$ and $i=1$.

split to $2^{w}$ vertices. Therefore, the base mesh has $s \times 2^{w}=2^{i+w}$ vertices. We divide these vertices into $s$ chunks and let the vertices split from a common ancestor to be in the same chunk. For each of these vertices, we also add its descendants up to $d$ levels. Thus, a chunk comprises of $2^{w}$ subtrees of vertices, each of height $d$. The vertices in a chunk satisfy the following conditions: (i) their vertex IDs (binary form) have a common $i$-bit prefix; (ii) the bit length of their vertex IDs is in $[i+1, i+d]$.

Therefore, $s$ chunks are available to be requested when only the base mesh is received, and each chunk has $2^{w} \times\left(2^{d}-1\right)$ vertex splits. When a chunk is decoded, $2^{w+d}$ vertices will be generated.

We generate $2^{d}$ chunks from these $2^{w+d}$ vertices by putting the vertices which only differ in the last $w$ bits into a chunk. These vertices are the roots of the subtrees in the chunk. Then, again for each vertex, we put its descendants up to $d$ levels in the same chunk. Therefore, we now have a chunk hierarchy with $2^{w}$ root chunks and each chunk has $2^{d}$ children chunks. We call this chunk the parent chunk of these $2^{d}$ children.

All vertices in a chunk have a common ancestor, so we assign the vertex ID of this common ancestor as the chunk ID of this chunk (e.g. in Figure 3, the vertices in a chunk '000' are all children of vertex '000'). In other words, the chunk ID is the longest common prefix of the binary form of the vertex IDs of its members.

Figure 3 is a simple example with $i=1, w=1$, and $d=2$. The base mesh has 4 vertices: 00, 01, 10 and 11. After the base mesh is received, two chunks 0 and 1 become available. The vertex splits for vertices 00 and 01 , as well as vertices from 000 to 011 are inside the chunk 0 . When chunk 0 is decoded, eight new vertices from 0000 to 0111 are generated. They become the root vertices of four new chunks from chunk 000 to chunk 011.

The main advantage of hierarchical chunk structure is that it fits the dependencies among the vertex splits well. First, such packetization does not increase the dependencies among chunks because vertices in a chunk are independent of any vertex splits except those inside the ancestor chunks. Second, both the dependency among chunk IDs and the relation between chunk ID and vertex ID are implicitly coded with the vertex ID. If the vertex ID of the root vertex is $I_{v}$, then the chunk ID is $I_{c}=\left(I_{v}>>d\right)$, and the ID of the parent chunk is $I_{p}=\left(I_{c}>>d\right)=\left(I_{v}>>2 d\right)$. Since the vertex ID itself can be deduced as introduced in Section 4, we implement this hierarchical system without any extra cost.

Moreover, since the vertices in a packet are all children of a common ancestor, they are connected and are typically close to each other. Therefore, if one of the root vertices is needed by a peer, other root vertices are likely needed as well. Hence, it is reasonable to put them into one chunk.

With this packetization, a peer that needs some vertex splits, can deduce the IDs of the chunks to request from the vertex IDs. Some 
invisible vertex splits may be received, but because vertices inside a chunk are nearby each other, they may be visible soon anyway when this peer changes viewpoint.

We now describe how to determine the values of $i, w$ and $d$. Suppose a chunk can carry about $n$ vertex splits after compression. Therefore we have

$$
2^{w} \times\left(2^{d}-1\right)<n
$$

Choosing a large $w$ can improve the quality more quickly since typically the vertex splits in low levels contribute more to the mesh quality, but it increases the possibility of including many invisible vertices. In our implementation, each chunk is the size of one packet, although we can easily generalized to larger chunk. Since about 300 vertices can fit into an IP packet (i.e. $n=300$ ), We choose $w=4$ and $d=4$, so a total of 240 vertex splits are in one chunk (if the progressive mesh is completely balanced).

After deciding the value $w$, value of $i$ can be decided based on the size of base mesh. Certain number of vertices are needed in the base mesh to ensure the minimal quality, and we know that the base mesh has $i \times 2^{w}$ vertices. So we can choose a proper $i$ value to satisfy the quality of base mesh. In our implementation, we choose $i=1024$. Thus, the base mesh has 16382 vertices.

\section{CONTENT DISCOVERY}

We now consider how a peer can discover other peers can provide a given chunk. We first consider distributed hash table (DHT) and gossip-based techniques, commonly used in file sharing and video streaming, and discuss why they are unsuitable for our application. We then describe a simple centralized scheme that works reasonably well, followed by a more complex scheme that exploits the hierarchical structure in the data, trading off the number of messages processed by server with response time and control overhead.

DHT, a well-known technique for content discovery, hashes a chunk to a peer, which maintains a directory of peers storing the chunk. DHT, however, is expensive to maintain and causes huge control overhead under high churn rate. As mentioned, short session time (typically several minutes) causes high churn rate in mesh streaming system. Moreover, DHT generally takes $O(\log n)$ hops to lookup, too long for the application we have in mind. We therefore preclude DHT in our solution.

Another common solution is gossip-based protocol, in which, peers exchange a bit-vector to notify each other which chunk it is holding on to. This approach is very flexible, but it does not fit well in P2P mesh streaming, either. The bit-vector is large due to small chunk size in our system, causing high control overhead. Second, due to short session times, much bandwidth is wasted in exchanging obsolete bit-vectors.

Compared to using DHT and gossip-based methods, maintaining a centralized lookup directory at the server has two advantages. First, the lookup is only a single hop. Further, the server can monitor peers' states and detect peer failures more quickly than in decentralized P2P systems, reducing the influence of churn. For these reasons, we study a $\mathrm{P} 2 \mathrm{P}$ mesh streaming system with centralized lookup and evaluate its performance.

\subsection{P2P With Centralized Lookup}

We first consider one of the simplest ways of supporting content discovery, using a central lookup server that maintains the information of providers for each chunk of the mesh. The server maintains a list of peers who have downloaded each chunk. A peer $p$ who needs a chunk $i$ contacts the server. The server chooses a peer who has chunk $i$ as provider and inform $p$ (see Figure 4 (a)). Peer $p$ informs the server after downloading chunk $i$, and is then added to

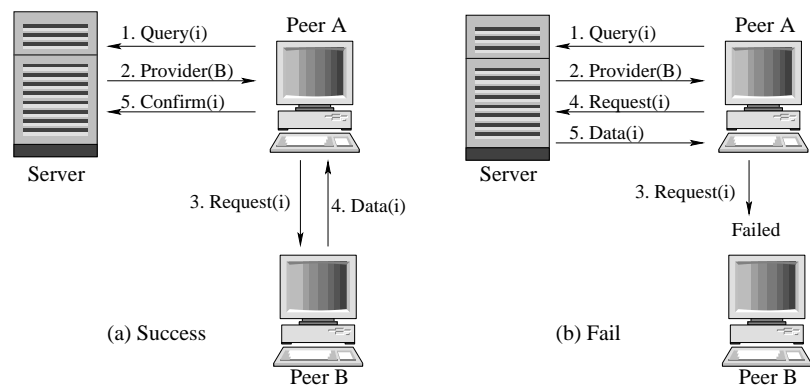

Figure 4: Centralized Lookup.

the list of peers with chunk $i$. The server becomes the provider for a chunk $i$ when there is no provider available for $i$ or when $p$ fails to receive a chunk from the given provider (e.g., if the provider has left or failed) (see Figure 4 (b)).

To keep the list of peers updated in an environment with high churn rate, the server monitors the list of providers. In our implementation, peers periodically (every 5 seconds in our implementation) send heart beat messages to the server, and the server "forgets" peers who have not been heard from after a certain period (15 seconds in our implementation).

The centralized lookup is suitable for a small or middle-scale P2P mesh streaming system. First, a query only costs two packets in terms of control overhead and one RTT in terms of delay. Second, states are maintained in one reliable node (the server), removing the needs of synchronizing states among peers. Third, the server, maintaining all states, can employ better algorithms to determine the provider to improve the performance. For example, topology-aware provider selection can reduce the response time and inter-ISP traffic; load- and capability-aware provider selection can balance the load among peers and improve fairness.

Nonetheless, the centralized lookup is not scalable to large number of peers, as provider monitoring and selection incur server overhead. Every request still causes a small packet to be sent from the server. We only decrease the size of outgoing packets, not the quantity. When the cost in handling requests is expensive (e.g. when topology-aware provider selection and load balancing is considered), the CPU becomes the bottleneck. For example, for a server with $100 \mathrm{Mbps}$ outgoing bandwidth and four $2 \mathrm{GHz}$ CPUs, assuming the outgoing packet size is 64 bytes, then the transmission delay is only $4.92 \mu \mathrm{s}$. If handling a request requires more than 40000 clock cycles, CPU becomes the bottleneck rather than the network bandwidth. Therefore, to further increase the scalability, we have to reduce the number of requests and the cost in handling requests.

To address this weakness, we propose a hierarchical P2P lookup approach, retaining most advantages of centralized lookup without introducing much overhead. We introduce this approach next.

\subsection{Hierarchical P2P Lookup}

In our hierarchical P2P lookup approach, a set of peers, called a group, is associated with each chunk of the mesh. A group $G_{i}$ contains only peers that have already received chunk $i$. Each group $G_{i}$ has a leader, denoted $l_{i}$, which acts similarly to the server in the P2P mesh streaming system with centralized P2P lookup we introduced above. The members act as the providers for the chunk and are monitored by the leader. Since the group and the chunk have a one-to-one mapping, the leader of group $G_{i}$ is also called the leader of chunk $i$.

Assume for now that a peer knows the group leader $l_{i}$ given $i$ (we will show how this is done later). When a peer $p$ needs chunk 
$i$, it contacts $l_{i}$ to join group $G_{i}$. The group leader $l_{i}$ then selects a provider from the members of $G_{i}$ and informs $p$ of the identity of this provider. The peer $p$ then contacts the provider for the chunk. Once $p$ receives the requested chunk from the provider, it informs $l_{i}$ and becomes a member of $G_{i}$.

Group leader acts as a provider when no other member exists. But, unlike the centralized lookup approach, if peer $p$ fails to receive chunk $i$ from a provider, it requests the chunk from the server rather than the group leader. The rationale here is that the server is more reliable than a group leader, so the response time can be guaranteed.

Note that one peer can belong to multiple groups. But a peer should not lead multiple groups simultaneously, (i) to ensure the peer has enough capacity to serve requests for one chunk, and (ii) to avoid a single peer failure affecting multiple groups.

Because a leader is also a peer, neither reliable nor having a wellknown, fixed, address, two questions need to be answered: (i) how each peer knows the leader of a given group; (ii) how to deal with the leader failure. We explain our solutions in following sections.

\subsubsection{Leader Hierarchy}

Leaders inherit the hierarchical structure of chunks, due to the one-to-one mapping between groups and chunks. The leaders form a tree, mirroring the structure of chunks. We use the common terms leaf, root, parent, and child to describe leaders and their relationship in a self-explanatory way. Further, we consider the server as the parent of all root leaders.

Due to the dependency among the chunks, children of a chunk $i$ is only useful after $i$ is received. Hence, it is natural for a leader to supply the information of its children to its members. When a peer joins the group $G_{i}$, in addition to the information of provider $s_{i}$, the leader $l_{i}$ also returns the information of its children. By exploiting the hierarchical property of the chunks, the leader information is obtained progressively without any query.

\subsubsection{Leader Assignment and Replacement}

In this section, we explain how leaders are assigned and how a failed leader is replaced. Initially, no peer exists in the system, and the server behaves as the default leader of all chunks. When a non-leader peer $p$ joins a group $G_{i}$ led by the server, the server assigns $p$ as the leader of $G_{i}$. The server will not assign a peer as a leader if this peer is leading another group. When there are many candidates, how the server selects leaders is an interesting topic for further research. In our current implementation, the server only chooses peers with enough uploading bandwidth (larger than $512 \mathrm{Kbps}$ ) as leaders.

Since leader assignments and replacements are done by the server, the server knows the current leader hierarchy. When assigning a peer $p$ as the leader of group $G_{i}$, the server also include information about $p$ 's parent and children in the tree. The new leader then notifies its parent and all its children of its new role.

To maintain the tree of leaders, a parent monitors all its children via heart beat messages. Failure of a child is reported by the parent to the server, which then chooses one non-leader peer from the peers who newly joined the system (see Figure 5(b)). The server maintains a list of recent, non-leader peers who query the server for root leaders initially. If no such peer is available, the server becomes the leader itself.

The leader replacement information is disseminated to the parent and children of the leader when the new leader notifies its role. The parent leader in turn notifies its own members of this leader changes. So a member in the parent group of $G_{i}$ will always know the updated leader of $G_{i}$.
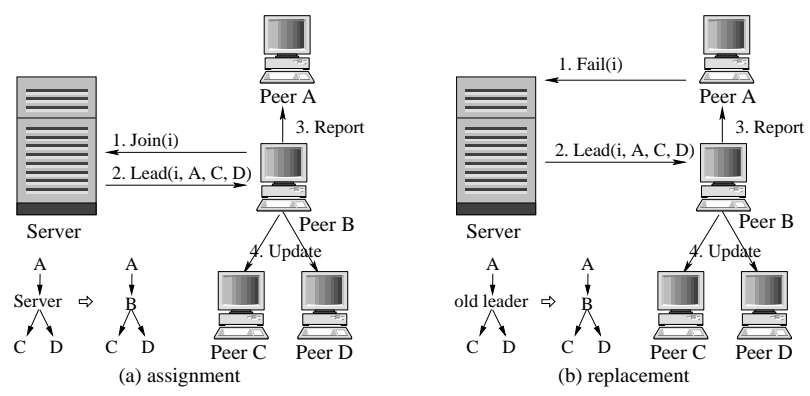

Figure 5: (a) Peer B is assigned a leader. (b) An old leader is replaced by peer $B$.

It is possible that a peer may contact a failed leader and experience timeout. If a peer receives new leader updates from the server, it contacts the new leader; otherwise it requests the chunk from the server and reports the failure.

\subsubsection{Reducing Monitoring Overhead}

A peer becomes a member of group $i$ when it receives chunk $i$. For a popular chunk $i$ (such as the root), many members may exist. Monitoring and maintaining a large number of members is expensive for the leader. Furthermore, after receiving many chunks, a peer belongs to many groups, leading to high control overhead. This problem can be easily solved by restricting the group size and the number of groups a peer belongs to.

We reduce the number of group a peer belongs to by the following rule: if a peer $p$ obtained two chunks $i$ and $j$, and $i$ is an ancestor of $j$, then $p$ only belongs to $G_{j}$.

By following the above rule, as a peer progressively obtains more and more chunks, it joins groups that are lower down the hierarchy and leaves the higher groups. Note that a peer leaves a group as long as it obtains any of the children chunks, not necessarily all. At a later time, a peer may want to download a child chunk $i$ of a group it has left (perhaps due to a change of view point). But, since a peer is no longer in the parent group of the chunk $i$, it may not have up-to-date information about the leader of $i$.

To avoid this, a group leader propagates updates about its children down to its sub-tree. As a result, a peer always knows its leader, the leader's ancestors, and the immediate children of the leader's ancestors. Knowing the children of the leader's ancestors allows a peer to download any children chunk through the leaders.

This solution raises another problem. If a peer belongs to multiple descendants of a group $G_{i}$, it may receive duplicate leader updates, leading to unnecessary messages. Such duplicates can be removed by simply having this peer to explicitly register it to the leader closest (in terms of hops count in the leader hierarchy) to $l_{i}$ for leader updates of $G_{i}$. This peer will not receive leader updates of $G_{i}$ from other leaders since leaders only send its members the messages they have registered.

For example, as shown in Figure 6, a peer is a member in both group 00000 and 0000300 . It has already received chunk 0, 000, and 00003 before, but has left those groups. Since it may join group 001, 002, and 003 in the future, it needs the leader updates from group 0. Group 00000 and 0000300 are both descendant groups of group 0 , but 00000 is closer to group 0 , so this peer registers to the leader of group 00000 for updates from group 0. Similarly, it registers to the leader of group 00000 for updates from group 000 and registers to the leader of group 0000300 for updates from group 00003. This method ensures that the leader updates will be received with the minimum delay and without duplication. 


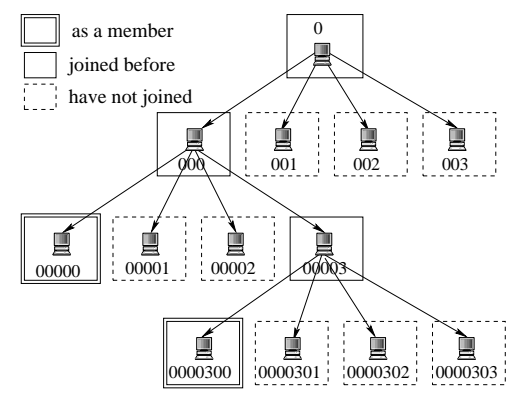

Figure 6: This peer will register in Group 00000 for leader updates from group 0 and 000 , and it will register in Group 0000300 for leader updates from group 00003.

Note that only non-leader members may leave a group. To keep the leader hierarchy stable, the leader always stays in the group until it fails or the server assigns a new leader for this group.

\subsubsection{Discussion}

In the P2P system with hierarchical P2P lookup approach, each group behaves like a small $\mathrm{P} 2 \mathrm{P}$ system with centralized lookup approach. First, most provider selection algorithms that can be used in centralized lookup approach, such as topology-aware and load balancing, can also be used in the hierarchical P2P lookup approach. Second, providers are also monitored, so the failure rate of requests is low even with high churn rate. Third, query for a provider can be finished within 1 RTT in most cases. In short, most of the advantages of the centralized lookup approach are retained.

Compared with the centralized lookup approach, the management overhead of the server in hierarchical P2P lookup approach is significantly reduced. In the stable stage, most group leaders are peers, so the server only monitors the leaders of the top chunks instead of all the peers in centralized P2P system. In addition, in hierarchical P2P lookup approach, the server is also responsible for replacing the failed leaders, and thus maintains the leader hierarchy at a small overhead. First, the number of leaders only relates to the number of chunks and thus is independent of the number of peers. Second, the rate of replacing leaders only relates to the leaving rate of the peers, which is typically small compared with the total number of peers. Thus, the management overhead is significantly smaller and the server is more scalable to a large number of peers compared with the centralized lookup approach.

The hierarchical P2P lookup approach, however, increases the control overhead and response time. It may also cause more chunks to be provided by the server. First, more control messages are needed to maintain the leader hierarchy, to propagate leader updates down to sub-trees, and to exchange heart beat messages between parent and children. Second, average response time increases since leaders may fail or leave. A peer who fails to contact a leader needs an additional RTT (to server) before receiving a chunk. Third, leader failure causes the server to provide more chunks. Another reason is that some valid providers may not be used after they leave a group, which will not happen in the centralized lookup approach.

In the next section, we further evaluate the centralized and hierarchical P2P lookup approaches by simulations.

\section{EXPERIMENTAL RESULTS}

In this section, we present the experimental results to evaluate the performance of the two lookup approaches we studied: centralized lookup and hierarchical P2P lookup.

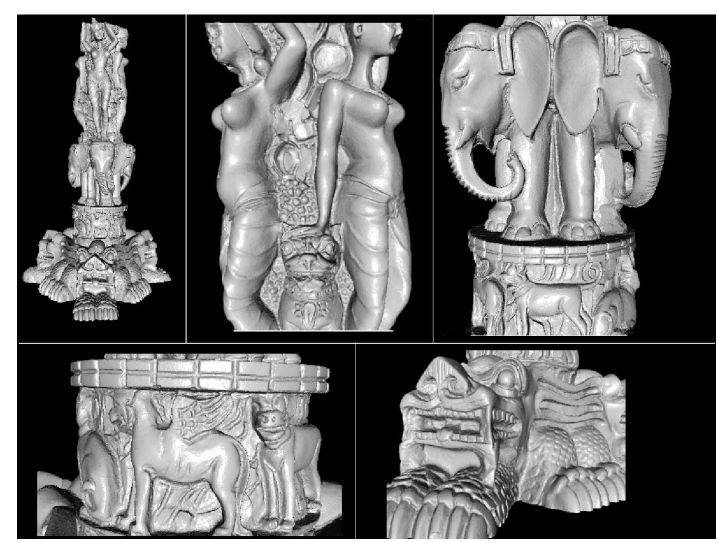

Figure 7: The Thai Statue. The top left is the whole mesh, and the rest is the closeup of some parts of it. Original mesh courtesy of Stanford Computer Graphics Laboratory.

\subsection{Experiment Setup}

We developed two systems to support the experiments: (i) a receiver-driven client and server implementing view-dependent streaming of progressive meshes used to collect and generate traces; and (ii) a simulator that replays the traces generated and simulates largescale P2P mesh streaming.

The client-server system is programmed in $\mathrm{C}++$ based on OpenGL and OpenMesh ${ }^{2}$. In our experiments, we use the Thai Statue from Stanford Computer Graphics Laboratory ${ }^{3}$, which has 5 millions vertices and takes up 22.5 MB after being compressed with our encoding method. The mesh is packetized into 147897 chunks following the approach introduced in Section 5. We choose this mesh since details exist all over the statue, so users may have interest in different positions and levels of details (see Figure 7).

We collected 60 traces from 37 students from our university. The session length of these traces ranges from 9 seconds to 380 seconds. When collecting the traces, the streaming bandwidth is set to 320 Kbps, with a client-server RTT of 400 millisecond.

To simulate many users, we generated 4000 random traces after analyzing the collected user behavior traces, following the same distribution of the session length (log-normal with $\mu=18.23, \sigma=$ 0.754 in $\mu \mathrm{s}$ ), inter-action time interval (generalized extreme value distribution with $\mu=266,370, \sigma=199,870$, and $\xi=0.51$, in $\mu \mathrm{s}$ ), and the probability of selecting an action (zoom, pan, or rotate). We replay these generated traces to obtain the sequence of chunk requests and use the latter in the simulation.

The P2P simulator is a discrete-event simulator based on OM$\mathrm{NeT}++$. In our simulator, peers follow a Poisson arrival model. Each peer randomly selects a trace from 4000 random traces we generated and leaves the system at the end of the trace. Our experiment lasts for 320 seconds, and the period from $300 \mathrm{~s}$ to $320 \mathrm{~s}$ is seemed as the stable stage (approximately). We choose the arrival rate $(\lambda)$ as 20,40,60, and 80 . During the stable stage, the number of online peers are around 1720, 3550, 5200, and 6920, respectively.

The end-to-end delay between two peers (including server to peers) are taken from the data collected from the Meridian [4] project. The original data is a $2500 \times 2500$ matrix recording the pair-wise delay between 2500 DNS servers. We assign each peer and the server a random DNS server and assume that the end-to-end de-

\footnotetext{
${ }^{2}$ http://www.openmesh.org

${ }^{3}$ http://www-graphics.stanford.edu/data/3Dscanrep/
} 

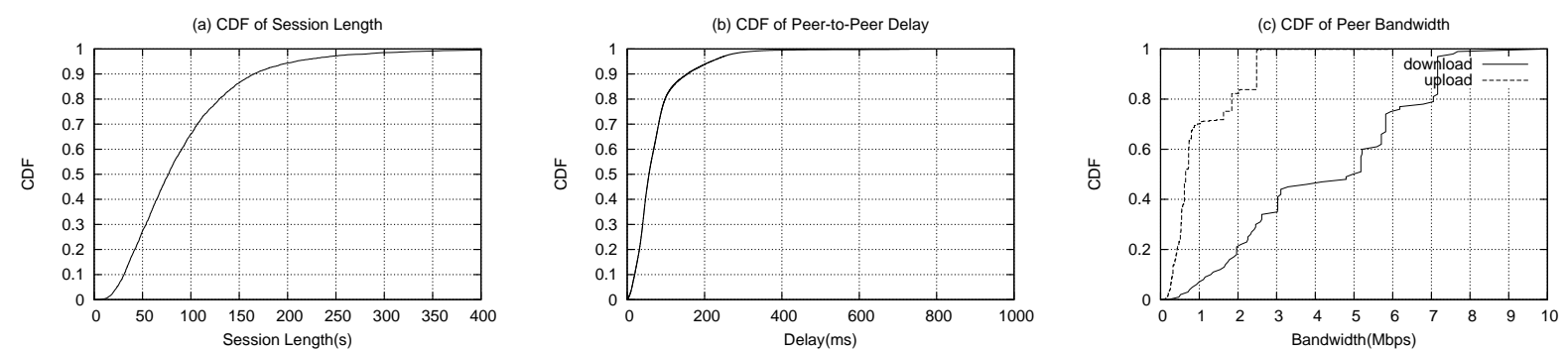

Figure 8: $\mathrm{CDF}$ of the session length $($ mean $=91.4 \mathrm{~s})$, peer-to-peer delay $($ mean $=75.8 \mathrm{~ms})$, and peer bandwidth $($ download mean $=$ 4292.8Kbps, upload mean $=1023.0 \mathrm{Kbps}$ ) used in our simulation.

lay of two peers equals the delay between their DNS servers (see Figure 8(b) for the CDF of end-to-end delay).

The downloading and uploading bandwidth of peers are randomly selected from the data reported by DSLReports.com ${ }^{4}$, which records and updates daily the access speed of users over the world. We use 21,206 samples, collected on 14 April 2009. Figure 8(c) shows the CDF of the downlink and uplink bandwidth.

In the following sections, we evaluate our P2P design in terms of server overhead, incoming message rate of the server, control overhead, and average response time.

\subsection{Server Overhead}

In this section, we measure the server overhead in three forms. First, we measure the outgoing data rate of the server. Second, we measure the ratio of the server's outgoing data rate to the total chunk size received by the peers. It is a relative server overhead compared with the client-server model based system. Third, to evaluate how busy a server is in handling incoming messages, we measure the incoming message rate of the server to indicate the server overhead in handling incoming requests.

The outgoing data rate is shown in Figures 9(a) and (b). Figures 9(a) shows that the server outgoing data rate in the client-server model exceeds $1 \mathrm{Gbps}$ when $\lambda$ exceeds 40 . It indicates that the client-server model cannot scale well without increasing the number of servers and outgoing bandwidth, so we will not consider the client-server model in further comparisons.

Figures 9(c) and (d) show the relative server overhead. The relative server overhead of the hierarchical P2P lookup is slightly larger than that of the centralized lookup in our implementation. The main reason is that more chunks are provided by the server in hierarchical P2P lookup than that in centralized lookup, as we will discuss in the section about response time.

The server incoming message rate of the two designs are shown in Figures 9(e) and (f), where we can see that the hierarchical P2P lookup reduces the number of incoming messages by around $80 \%$. Moreover, the incoming message rate increases more slowly with $\lambda$ in hierarchical P2P lookup, indicating that the CPU overhead of the server (to handle the messages) also increases more slowly.

Note that in these two designs, the server responses to incoming requests are different. The responses are leader information (for failed joins) and chunks (for failed requests) in hierarchical $\mathrm{P} 2 \mathrm{P}$ lookup approach, but are mainly provider information in centralized approach. Retrieving a chunk or leader information is typically cheaper than deciding the best provider, especially when topology awareness and load balancing are considered. Hence hierarchical $\mathrm{P} 2 \mathrm{P}$ lookup not only reduces the incoming message rate, but also reduces the handling overhead per request to the server.

\footnotetext{
${ }^{4}$ http://www.dslreports.com/archive
}

\subsection{Control Overhead}

In this section we examine how much network bandwidth is used by control messages. We define control overhead as the ratio of the size of control messages to the size of chunks received by all peers during one second. Figures $9(\mathrm{~g})$ and $(\mathrm{h})$ show that hierarchical P2P lookup has higher control overhead than centralized lookup. The higher overhead is caused by maintaining the leader hierarchy and propagating leader updates. Moreover, in hierarchical P2P lookup, peers may belong to multiple groups, leading to more messages used in monitoring. Nonetheless, the control overhead of both systems are within an acceptable range.

\subsection{Response Time}

Another important metric to evaluate the P2P mesh streaming system is the response time. The response time for each chunk request is obtained by subtracting the receiving time of the chunk by the sending time of the corresponding request. Since no strict order in receiving chunks is required in mesh streaming, the response time of a single request is not as important as that in audio and video streaming. Instead, we emphasize on the average response time of requests sent in a second, which is the average value of all the response times of chunk requests in a second.

Figures 9(i) and (j) show that the hierarchical P2P system has higher average response time. The higher response time is caused mainly by two reasons. First, unlike providers, only one leader exists for one chunk. Leader failures cause many peers to spend one more round trip to obtain chunks. It is possible to use multiple leaders in a group in the future to reduce this effect. Second, a leader in hierarchical P2P lookup approach does not have the information of all the providers as the server in centralized lookup, and congestion may happen due to limited supply ability, causing request failures and higher response time.

The response time of hierarchical $\mathrm{P} 2 \mathrm{P}$ approach, however, is still under 1 second, which according to our experience, does not significantly affect users' experience. When a user interacts with the mesh (e.g., rotate), the renderer responds almost immediately. The response time of chunk requests only affects how fast the mesh quality improves after users change their view points.

\subsection{Summary}

The results above indicate that both designs work well under an environment with heterogeneous peers, asymmetric bandwidth, and high churn rate. Compared with a client-server design, the server outgoing bandwidth is reduced by more than $90 \%$. Hierarchical P2P lookup also reduces $60 \%$ of incoming messages to the server, but generates only around $10 \%$ of control overhead when the number of peers is large. The average response time of both systems is below a second and does not affect the user experience 
(a) Server Outgoing Datarate vs Time
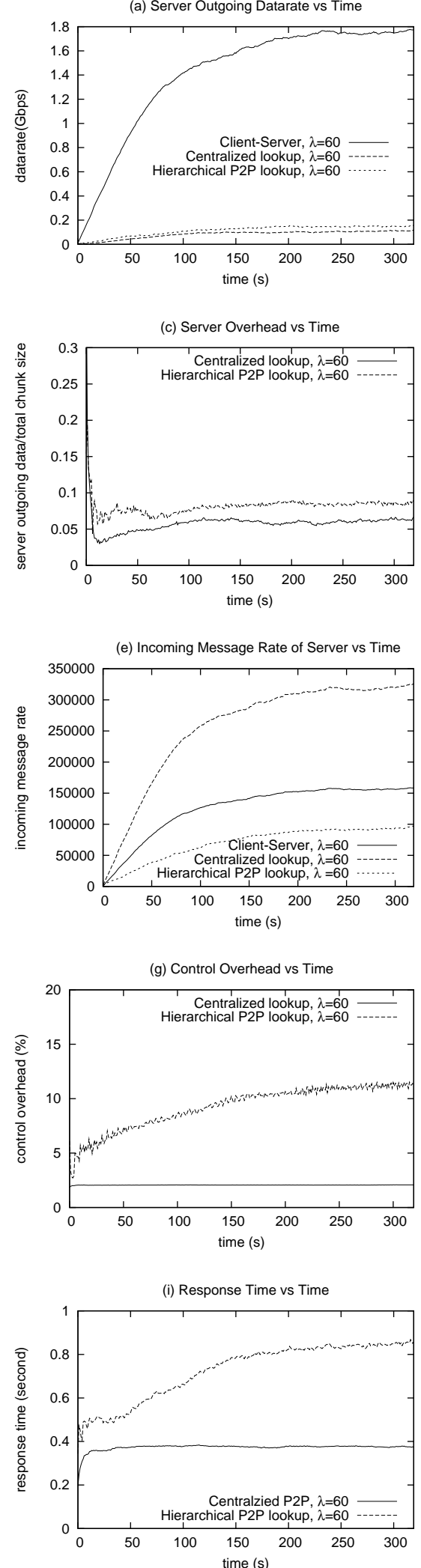
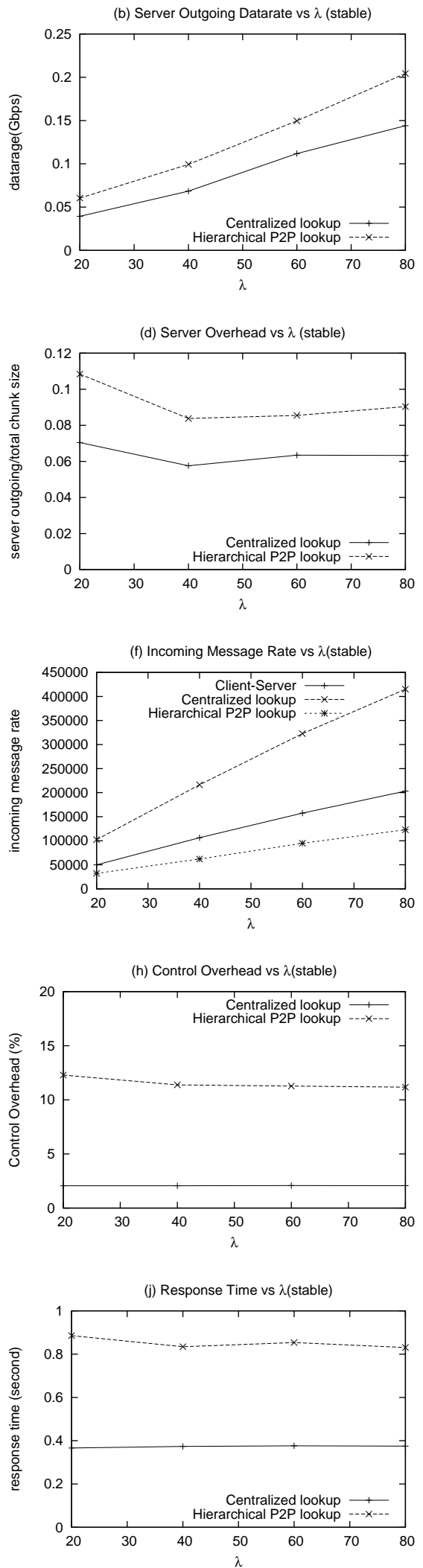

Figure 9: Comparison between centralized lookup approach and hierarchical P2P lookup approach. The left column indicates how the results change with time, and the right column how the results change with the arriving rate of peers. The value in stable stage is averaged from $t=300 \mathrm{~s}$ to $t=320 \mathrm{~s}$. 
due to the progressive rendering nature of the mesh. Further, as the number of peers increases, the control overhead and response time remain relatively stable.

\section{CONCLUSION AND FUTURE WORK}

This paper investigates into the problem of $\mathrm{P} 2 \mathrm{P}$ view-dependent, progressive mesh streaming, and studied two important components of the problem - chunking and content discovery. We find that in P2P mesh streaming, peers need to keep finding new chunk providers, increasing the control overhead. Furthermore, the short session length of peers increases the churn rate. We considered these unique characteristics of mesh streaming and explored two content discovery schemes. We found that centralized lookup works well with these challenges. To further reduce the CPU overhead of the server, a hierarchical P2P lookup approach can be used to move the lookup service to selected peers.

Further research can be done on other aspects of $\mathrm{P} 2 \mathrm{P}$ mesh streaming. First, authentication is needed to detect malicious tampering of the mesh by peers. Second, the user pattern in viewing the mesh could be exploited for pre-fetching. We plan to pursue these research issues next.

Acknowledgement: This project is supported by National University of Singapore Academic Research Fund R-252-000-306-112.

\section{REFERENCES}

[1] G. Al-Regib and Y. Altunbasak. 3TP: An application-layer protocol for streaming 3D models. IEEE Transactions on multimedia, 7(6):1149-1156, December 2005.

[2] G. Al-Regib, Y. Altunbasak, and J. Rossignac. Error-resilient transmission of 3D models. ACM Trans. Graph., 24(2):182-208, 2005.

[3] P. Alliez and M. Desbrun. Progressive compression for lossless transmission of triangle meshes. In Proceedings of SIGGRAPH '01, pages 195-202, Los Angeles, CA, August 2001.

[4] A. S. Bernard Wong and E. G. Sirer. Meridian: A lightweight network location serive without virtual coordinates. In proceedings of SIGCOMM'05, Philadelphia, PA, August 2005.

[5] M. Castro, P. Druschel, A.-M. Kermarrec, A. Nandi, A. Rowstron, and A. Singh. SplitStream: High-bandwidth content distribution in cooperative environments. In Proceedings of SOSP'03, pages 298-313, Sagamore, NY, October 2003.

[6] R. Cavagna, C. Bouville, and J. Royan. P2P network for very large virtual environment. In Proceedings of ACM VRST'06, Limassol, Cyprus, November 2006.

[7] Z. Chen, J. F. Barnes, and B. Bodenheimer. Hybrid and forward error correction transmission techniques for unreliable transport of 3D geometry. Multimedia Systems, 10(3):230-244, March 2005.

[8] W. Cheng and W. T. Ooi. Receiver-driven view-dependent streaming of progressive mesh. In Proceedings of NOSSDAV'08, Brauschweig, Germany, May 2008.

[9] W. Cheng, W. T. Ooi, S. Mondet, R. Grigoras, and G. Morin. An analytical model for progressive mesh streaming. In Proceedings of MULTIMEDIA '07, pages 737-746, Augsberg, Germany, September 2007.

[10] Y. Chu, S. Rao, and H. Zhang. A case for end system multicast. In Proceedings of SIGMETRICS'00, pages 1-12, Santa Clara, CA, June 2000.
[11] Y. Cui, B. Li, and K. Nahrstedt. oStream: asynchronous streaming multicast in application-layer overlay networks. IEEE journal on selected areas in communications, 22(1):91-106, January 2004.

[12] T. T. Do, K. A. Hua, and M. A. Tantaoui. P2VoD: providing fault tolerant video-on-demand streaming in peer-to-peer environment. In Proceedings of IEEE ICC'04, volume 3, pages 1467-1472, Paris, France, June 2004.

[13] A. F. Harris (III) and R. Kravets. The design of a transport protocol for on-demand graphical rendering. In Proceedings of NOSSDAV'02, pages 43-49, Miami, FL, May 2002.

[14] H. Hoppe. Progressive meshes. Computer Graphics, 30(Annual Conference Series):99-108, August 1996.

[15] H. Hoppe. View-dependent refinement of progressive meshes. In Proceedings of SIGGRAPH '97, pages 189-198, Los Angeles, CA, August 1997.

[16] S.-Y. Hu, T.-H. Huang, S.-C. Chang, W.-L. Sung, J.-R. Jiang, and B.-Y. Chen. FLoD: A framework for peer-to-peer 3D streaming. In Proceedings of IEEE INFOCOM'08, Phoenix, AZ, April 2008.

[17] J. Jannotti, D. Gifford, K. L. Johnson, M. F. Kaashoek, and J. W. O. Jr. Overcast: reliable multicasting with on overlay network. In Proceedings of OSDI'00, pages 1-14, San Diego, CA, October 2000.

[18] J. Kim and S. Lee. Truly selective refinement of progressive meshes. In Proceedings of Graphics Interface 2001, pages 101-110, June 2001.

[19] H. Li, M. Li, and B. Prabhakaran. Middleware for streaming 3D progressive meshes over lossy networks. ACM Trans. Multimedia Comput. Commun. Appl., 2(4):282-317, 2006.

[20] N. Magharei and R. Rejaie. PRIME: Peer-to-peer receiver-driven mesh-based streaming. In Proceedings of INFOCOM'07, Anchorage, Alaska, 2007.

[21] V. N. Padmanabhan, H. J. Wang, and P. A. Chou. Resilient peer-to-peer streaming. In Proceedings of ICNP'03, page 16, Atlanta, GA, November 2003.

[22] S.-B. Park, C.-S. Kim, and S.-U. Lee. Error resilient coding of 3D meshes. In Proceedings of ICIP, volume 1, pages I-773-6, 2003.

[23] S.-B. Park, C.-S. Kim, and S.-U. Lee. Error resilient 3-D mesh compression. IEEE Transactions on Multimedia, 8(5):885-895, October 2006.

[24] D. Tian and G. AlRegib. On-demand transmission of 3D models over lossy networks. EURASIP Journal on Signal Processing: Image Communication, 21, June 2006.

[25] Z. Yan, S. Kumar, and C.-C. Kuo. Error-resilient coding of 3-D graphic models via adaptive mesh segmentation. IEEE Transactions on Circuits and Systems for Video Technology, 11(7):860-873, July 2001.

[26] Z. Yang, W. Wu, K. Nahrstedt, G. Kurillo, and R. Bajcsy. Viewcast: View dissemination and management for multi-party $3 \mathrm{~d}$ tele-immersive environments. In Proceedings of MULTIMEDIA '07, Augsberg, Germany, September 2007.

[27] W.-P. K. Yiu, X. Jin, and S.-H. G. Chan. Distributed storage to support user interactivity in peer-to-peer video streaming. In Proceedings of IEEE ICC'06, pages 55-60, Istanbul, Turkey, June 2006.

[28] X. Zhang, J. Liu, B. Li, and T.-S. P. Yum. CoolStreaming/DONet: A data-driven overlay network for efficient live media streaming. In Proceedings of INFOCOM'05, pages 2102-2111, Miami, FL, March 2005. 